

\title{
CHARACTERIZATION OF NANOPHASE MATERIALS BY X-RAY DIFFRACTION AND COMPUTER SIMULATION
}

\author{
J. A. EASTMAN and L. J. THOMPSON \\ Materials Science Division, Argonne National Laboratory, Argonne, IL 60439-4838
}

\begin{abstract}
$\mathrm{X}$-ray diffraction experiments on nanophase $\mathrm{Pd}$ have been performed with the primary goal of determining the nature of grain boundary structures in nanophase materials. A kinematical diffraction analysis has been developed to interpret $x$-ray $\theta-2 \theta$ data by comparing actual scans with scans produced by computer simulation. This simulation program has been used to explore the effects on diffracted intensity of a variety of microstructural and grain boundary structural parameters such as void concentration, grain size, grain boundary width, and changes in interplanar spacing and density in grain boundary regions. It has been found that a reasonable match to experimental data is produced by at least two model structures; in one, the material contains randomly positioned voids or vacancies, while in the other, the interplanar spacings in grain boundary regions are varied with respect to the spacings found in the grain interiors.
\end{abstract}

\section{INTRODUCTION}

Nanophase (ultrafine-grained) materials have recently been shown to possess a variety of interesting and novel properties [1-3]. The high fraction of atoms located within $0.5 \mathrm{~nm}$ of grain boundaries ( $\sim 50 \%$ for $5 \mathrm{~nm}$ grain size) means that grain boundaries are likely to be dominant in determining and controlling properties. Therefore, it is important to have an understanding of the nature of the atomic structure of the grain boundaries. Zhu et al. [4] interpreted $x$-ray $\theta-2 \theta$ data as indicating that grain boundaries in nanophase materials lack both short- and long-range order, and thus are fundamentaliy different in character than grain boundaries in conventional, coarser-grained material. They claim that a good match to experimental $\theta-2 \theta$ scans can only be obtained if atoms located within $1 \mathrm{~nm}$ of grain boundaries are relaxed in random directions from the normal sites that they would occupy if located far from a boundary. Thus, in their interpretation, the boundary structures in nanophase materials could be thought of as representing a new solid state structure.

In this paper, it will be shown that a lack of short- and long-range order in nanophase grain boundaries is not a requirement in order to interpret $\theta-2 \theta$ data. $\mathrm{X}$-ray diffraction $\theta-2 \theta$ observations from nanophase Pd will be described and interpreted using a model calculation developed to simulate $\theta-2 \theta$ diffraction scans from polycrystalline samples. Due to the brevity of this paper, only highlights of the work will be given. A more complete report is being prepared and will be published elsewhere.

\section{EXPERIMENTAL TECHNIQUES AND RESULTS}

Nanophase Pd having an average grain size of approximately $5 \mathrm{~nm}$ (and a log-normal grain size distribution) was produced by evaporating $\mathrm{Pd}$ wire $(99.997 \%$ purity) in $500 \mathrm{~Pa}$ of $\mathrm{He}$ (99.999\% purity) and then collecting and consolidating the resulting powders using pressures of $1.4 \mathrm{GPa}$ under vacuum conditions. The samples produced were $9 \mathrm{~mm}$ diameter discs with typical thicknesses of 100-300 microns. The density of the as-produced samples was determined by direct measurement of mass and volume and was found to be typically $70-80 \%$ of normal bulk density. 
$X$-ray $\theta-2 \theta$ scans were obtained in back-reflection mode using two diffractometer units, both of which use $\mathrm{Cu}$ radiation and a scintillation detector. One unit was equipped with a crystal monochromator on the detector side of the sample in order to eliminate the white background and $\mathrm{K}_{\beta}$ contributions. The other system did not have a monochromator, but was electronically gated to detect only $K_{\alpha}$ plus a small portion of the white spectrum. Peak breadths and shapes were observed to be the same for the two systems, but relative background levels were higher in the non-monochromated system. Because of this, only scans from the monochromated system have been used when determining peak-height-to-background ratios for comparison with calculated scans. Samples were mounted on glass slides with a small drop of epoxy for the x-ray experiments. Background contributions from the glass slide were unavoidable due to the small sample size. The magnitude of the unwanted background was estimated by comparing scans taken with and without samples mounted on the slide. For comparing actual and simulated scans, this spurious background contribution was then removed.

A typical $\theta-2 \theta$ scan from nanophase Pd is shown in Fig. 1(a). The normal Bragg peaks for fcc Pd are visible and there are no measurable peak shifts. Thus, there is no change in the lattice parameter as a result of the small grain size. The peaks were observed to be very symmetric about the Bragg angle as shown in Fig. 1(b). Significant intensity above background could be seen extending to angles much larger than twice the full-width at half-maximum intensity (FWHM).

The average grain size value calculated from the FWHM using the Scherrer equation was found to be 5-7 nm, in reasonable agreement with that obtained from transmission electron microscopy observations of nanophase Pd powder. A significant discrepancy was typically found for the (111) peaks though, with values calculated from this peak typically $30-50 \%$ larger than those calculated for the other peaks and observed by TEM. This discrepancy could be due to strain or to small numbers of relatively large (111)-oriented grains being present in the sample.

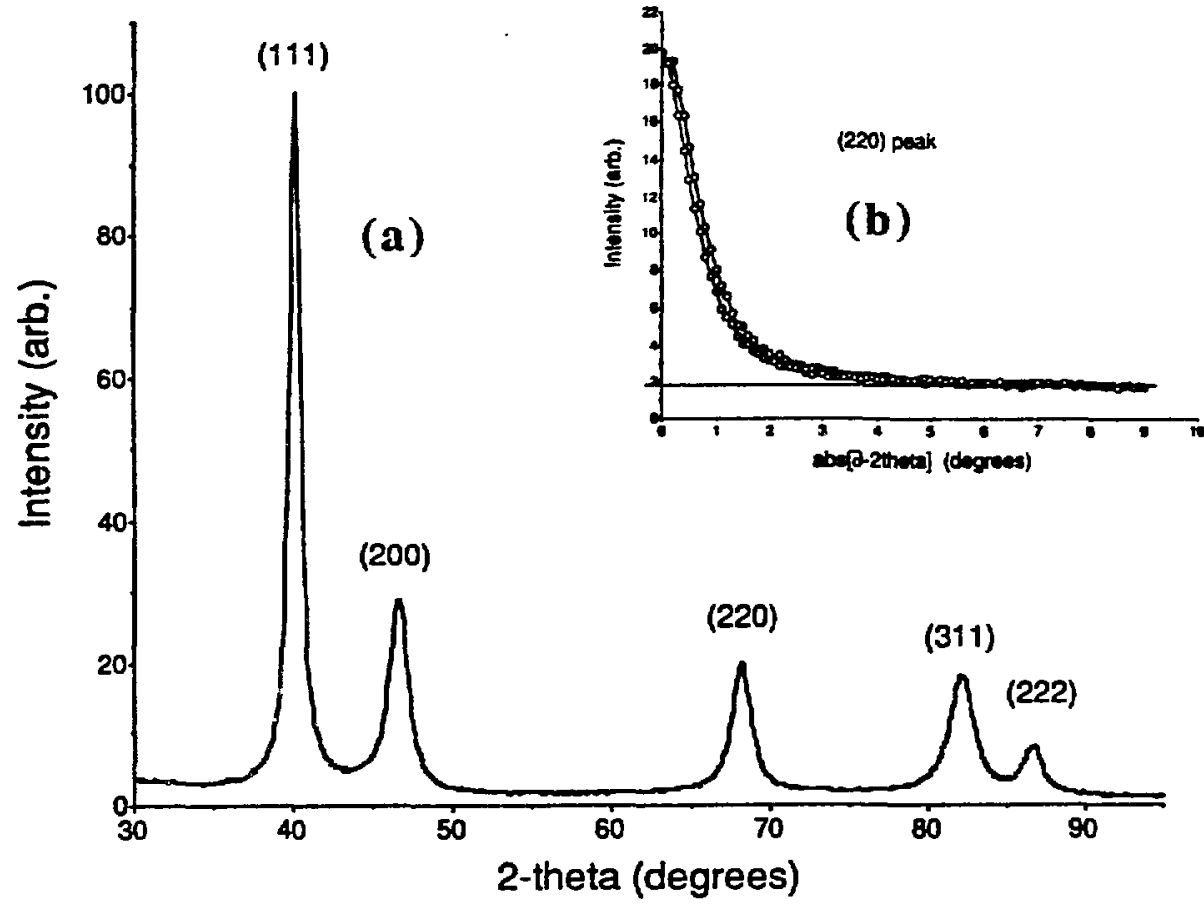

Figure 1. (a) $\theta-2 \theta$ scan from nanophase Pd having an average grain size of approximately 5 nm. (b)-(inset) Detail of the (220) Bragg peak from (a) showing the typical symmetric peak shapes observed. 


\section{MODEL DESCRIPTION AND COMPARISON WITH EXPERIMENT}

Diffracted amplitudes were calculated using the following form of the standard kinematical diffracted amplitude equation:

$$
A(s)=\sum_{\mathbf{n}} A_{0} f \exp \left[2 \pi i\left(s \cdot \mathbf{r}_{\mathbf{n}}\right)\right]
$$

where $A_{1}$ is the incident amplitude (set equa' to 1 since absolute intensities were not calculated), $f$ is the atomic scattering factor, $s$ is the scattering vector $(s=2(\sin \theta) / \lambda)$ and $\mathbf{r}_{\mathbf{n}}$ is the position vector of the nth scatterer. The diffracted intensity at a given angle $\theta$ is the amplitude at that angle multiplied by its complex conjugate. To compare simulated and actual scans, the simulated plots were multiplied by the Lorentz-polarization factor.

The calculation was simplified by realizing that $\theta-2 \theta$ scans are one-dimensional; i. e., the direction of the scattering vector is constant for all $\theta$. This means that it is only necessary to include the periodicities along a single direction in the calculation. Therefore, instead of having to calculate the amplitude contributions of several thousand atoms, it is necessary only to account for perhaps 20-50 atomic spacings along the scattering vector. This allowed the calculation to be done for a truly polycrystalline model consisting of several hundred grains rather than having to use a single "average" grain, as was done in the analysis of Zhu et al.[4].

In the simulation program, the grains were modeled as consisting of two parallel arrays of scatterers (atoms or planes of atoms) aligned along the direction of the scattering vector. One array represented the periodicities in the grain interior, while the other represented the periodicities in the grain boundary regions. The contribution of each array to the diffracted amplitude was weighted taking into account the grain size and grain boundary width. Each individual grain included in the present calculation was assumed to have either (111), (200), (220), (311) or (331) type planes oriented perpendicular to the scattering vector, corresponding to the five lowest-index type planes with non-zero structure factor in fcc materials. A random number generator was used to determine the position of the first plane in each grain. The relative numbers of each type of plane included were determined by their multiplicity factors. While the vast majority of grains in an actual nontextured sample would have a high index plane perpendicular to the scattering vector, the contribution of these grains to the scattering in the vicinity of the low-index Bragg reflections, where comparisons were made, is expected to be minimal.

The grain boundary structural parameters included in the simulation were the grain boundary width (corresponding to the region in which significant atomic relaxations occur), the average change in planar spacing in the grain boundary regions and the average density in grain boundary regions. The grain boundary region plane spacings and densities were held constant within a single grain, but varied from grain to grain within controlled limits. Other structural parameters included were the lattice parameter, the grain size (and grain size distribution) and the void or vacancy concentration within grains. Thermal vibrations have not yet been included in the calculation since the magnitude of these vibrations may differ from those in coarser-grained materials and the temperature experiments necessary to determine this have not been performed. While all of the above-mentioned parameters affect the simulated scans, describing the detailed effects of each of the parameters is beyond the scope of this paper.

A comparison of the observed $\theta-2 \theta$ scan in Fig. 1 with a simulated scan produced from a model consisting of approximately 500 "perfect" grains (no voids or changes in plane spacing in grain boundary regions) having an average grain size of $5.6 \mathrm{~nm}$ is shown in Fig. 2. The two scans were overlaid by scaling the intensities in order to match the background intensities well away from any Bragg peaks (the average values between $2 \theta=55$ and $60^{\circ}$ were used in scaling the plots). Comparing the two scans, it can be seen that the shape and location of the peaks are the same, but 


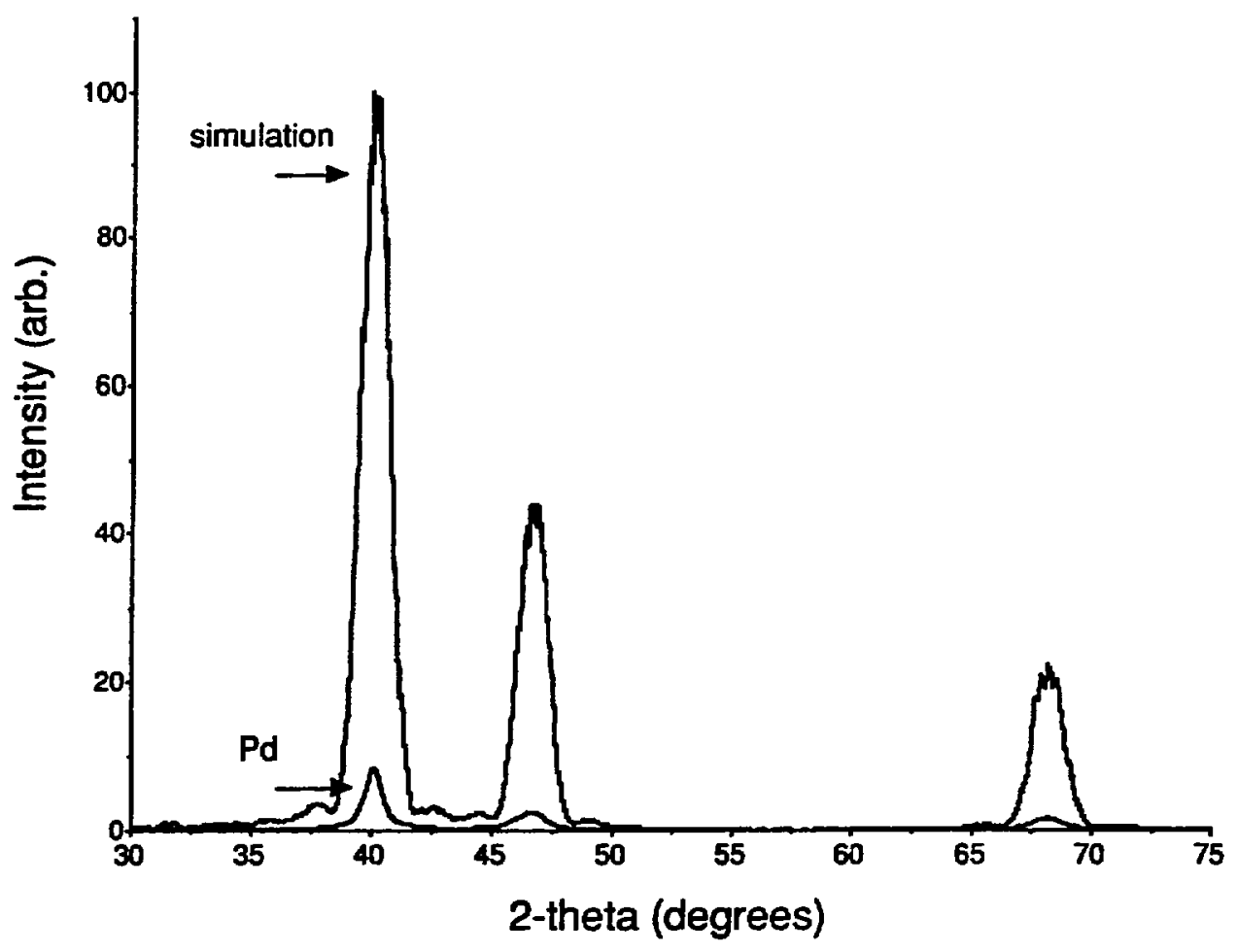

Figure 2. Comparison of $\theta-2 \theta$ scans from approximately $5 \mathrm{~nm}$ grain-sized nanophase Pd with a simulation calculated assuming no voids or changes in grain boundary plane spacings or densities. The simulated scan was calculated assuming a mean grain size equal to that of the $\mathrm{Pd}$, and a grain size distribution similar to that observed.

that the peak-height-to-background ratios are considerably higher in the simulated scan than in the actual scan (by a factor of 8-12x, depending on the peak measured). Although the background scattering from the sample holder was estimated and subtracted before making this comparison, this process is probably not without error. The relative magnitude of the background intensity in the simulated scan may also be somewhat underestimated due to the omission of high-index planes from the calculation. Thus, while it is believed that the experimentally observed peak-height-tobackground ratios in nanophase Pd are clearly lower than predicted by the simulation, the actual magnitude of the decrease may not be as large as that shown in Fig. 2. These observations agree qualitatively with those reported by Zhu et al. for nanophase Fe [4]. While they did not tabulate this information in their report, by comparing the actual and calculated scans shown in Fig. 7 of that work, it appears that at least a factor of 2-5x change was observed.

The effects of changes in plane spacing in the grain boundary regions are shown in Fig. 3. In this figure, the simulated scan produced from perfect $5.6 \mathrm{~nm}$ grains is overlaid with a scan produced from a model containing the same grain size and number of planes, but in which planes located within $0.5 \mathrm{~nm}$ of grain boundaries ( $1 \mathrm{~nm}$ boundary width) have densities of $60-100 \%$ of the bulk and plane spacings of $50-150 \%$ of those in the grain interiors (the density and plane spacing in the grain boundary regions is different in each individual grain). The displacements in this case are similar in magnitude to those used by Zhu et al. [4], but they are not in random directions. Thus, the grain boundaries can still be thought of as being ordered. The effect of these displacements is to reduce the peak-to-background ratios by a factor of $1.5-2 \mathrm{x}$, which is a smaller effect than was actually observed. The important point to recognize is that the peak-height-tobackground ratios are changed by changes in grain boundary plane spacing and density. It should be pointed out that atomic displacements as large as those used in this simulation are probably unlikely to occur, and no evidence for such large displacements has been observed in recent high 


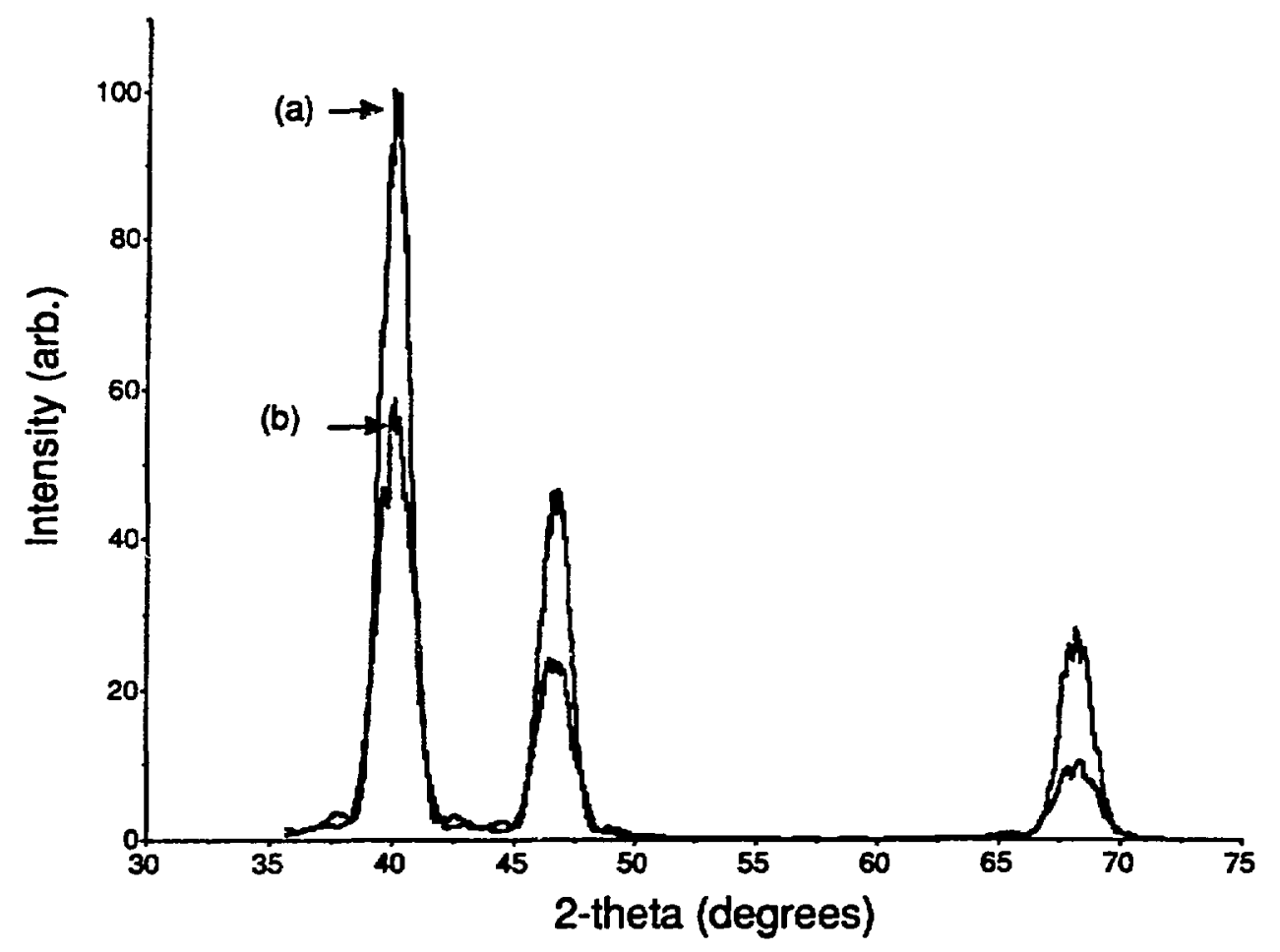

Figure 3. Comparison of two simulated $\theta-2 \theta$ scans. (a) is the simulated scan from Fig. 2, which assumes perfect grains, while (b) was calculated assuming the density within a $1 \mathrm{~nm}$, wide boundary region varies between $60-100 \%$ of bulk density and the plane spacings in these regions range from $50-150 \%$ of the plane spacings in the grain interiors.

resolution transmission electron microscopy studies [5]. The electron microscopy studies have also suggested that the boundary widths in nanophase $P d$ are closer to $0.5 \mathrm{~nm}$ than $1.0 \mathrm{~nm}$. Narrower boundaries would result in a smaller reduction in the peak-to-background ratios. Because the planes in this simulation had equal probabilities of having either increased or decreased spacings with respect to their normal bulk spacings, the peaks remained symmetric. It was observed that asymmetries were produced in the Bragg peaks if the average plane spacing in the grain boundary regions was not equal to the perfect crystal spacing. The form of the asymmetries resembles that seen in bicrystal studies [6].

A second means of producing smaller peak-to-background ratios was found by considering the effect of voids or vacancies within grains on the diffracted intensities. Fig. 4 shows a comparison of the scans predicted for a perfect crystals model with a model in which $20 \%$ of the atoms were removed from random sites within the grains. The intensities of the two scans were adjusted so that the effective total number of scatterers was constant. This model structure did not include any contributions due to changes in plane spacing in the regions near grain boundaries. It can be seen from this figure that the presence of vacancies or voids results in a significant reduction in the peak-to-background ratios. Thus, it is possible that voids are responsible for a major portion of the observed background intensity. It is not unreasonable to expect large void concentrations to be present within grains, since it is known that the individual grains produced by the gascondensation process are formed by the coalescence of small atomic clusters, and metal clusters themselves show evidence of containing significant concentrations of surface vacancies [7]. Large concentrations of fine scale voids are also believed to be localized in the boundary regions, since the nanophase samples are consolidated at room temperature. Thus, while it seems unlikely that atomic relaxations alone are responsible for the observed $\mathrm{x}$-ray intensities, it is possible that finescale voids or a combination of voids and atomic relaxations near grain boundaries can account for the observations. 


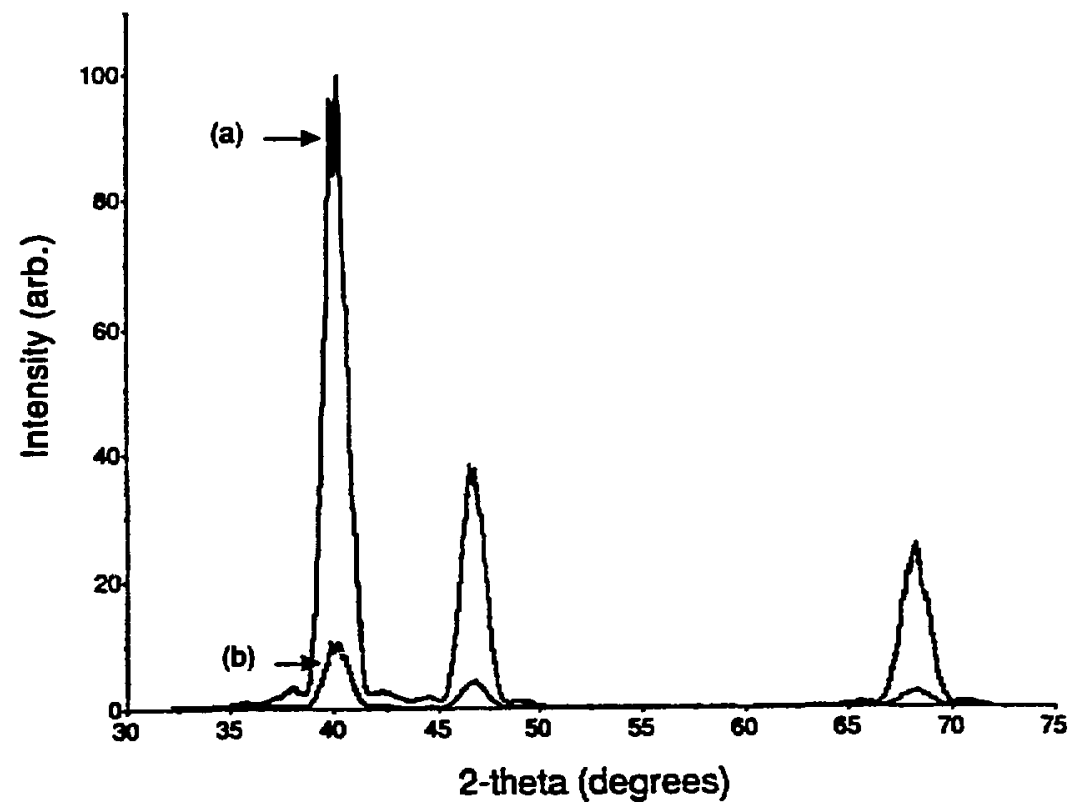

Figure 4. Comparison of two simulated $\theta-2 \theta$ scans. (a) is again the perfect grain simulation seen in Fig. 2, while (b) was calculated for a model containing $20 \%$ randomly located yoids or vacancies.

\section{CONCLUSIONS}

The main conclusions of the present investigations are: (1) Symmetric Bragg peaks with no peak shifts or extra peaks are observed in $x$-ray $\theta-2 \theta$ scans from nanophase Pd. (2) Peak-heightto-background ratios observed are smaller than those expected from simulations calculated for perfect grains containing no voids or atomic relaxations in grain boundary regions. Thus, diffuse background intensity is produced. The observed background intensities in the present work may contain artifacts, however, due to contributions from the sample holders used, and thus, better data are required before quantitative comparisons can be made. In addition, better knowledge of void concentrations within the materials is required. (3) Generation of diffuse background intensity such as that observed does not require relaxations of grain boundary atoms in random directions, as previously reported by $\mathrm{Zhu}$ et al [4]. Reasonable match with the observed $\theta-2 \theta$ scans could be produced by accounting for contributions from both the presence of voids and ordered atomic relaxations within grain boundary regions. Much work clearly still remains to be done in order to fully characterize the grain boundary structures present in nanophase materials.

This work was supported by the U.S. Department of Energy, BES-DMS, under Contract W-31109-Eng-38.

\section{REFERENCES}

1. R. Birringer, U. Herr and H. Gleiter, Suppl. Trans. Jpn. Inst. Met. 27, 43 (1986).

2. R. W. Siegel and J. A. Eastman, Mater. Res. Soc. Symp. Proc. 132, 3 (1989).

3. R. P. Andres et al., J. Mater. Res. 4, 704 (1989).

4. X. Zhu, R. Birringer, U. Herr and H. Gleiter, Phys. Rev. B 35, 9085 (1987).

5. G. J. Thomas, R. W. Siegel and J. A. Eastman, these Proceedings.

6. M. D. Vaudin, E. Burkel and S. L. Sass, Phil. Mag. A 54, 1 (1986).

7. P. A. Montano, G. K. Shenoy, E. E. Alp, W. Schulze and J. Urban, Phys. Rev. Lett., $\underline{56}$ (19), 2076 (1986). 